EPJ manuscript No.

(will be inserted by the editor)

Rapid Note

\title{
Mott transition of the $f$-electron system in the periodic Anderson model with nearest neighbor hybridization
}

\author{
K. Held ${ }^{\mathrm{a}}$ and R. Bulla \\ Theoretische Physik III, Elektronische Korrelationen und Magnetismus, Universität Augsburg, 86135 Augsburg, Germany
}

October 26, 2018

\begin{abstract}
We show analytically that, under certain assumptions, the periodic Anderson model and the Hubbard model become equivalent within the dynamical mean field theory for quasiparticle weight $Z \rightarrow 0$. A scaling relation is derived which is validated numerically using the numerical renormalization group at zero temperature and quantum Monte Carlo simulations at finite temperatures. Our results show that the $f$-electrons of the half-filled periodic Anderson model with nearest neighbor hybridization get localized at a finite critical interaction strength $U_{\mathrm{c}}$, also at zero temperature. This transition is equivalent to the Mott-transition in the Hubbard model.
\end{abstract}

PACS. 71.27.+a Strongly correlated electron systems; heavy fermions - 71.10.Fd Lattice fermion models (Hubbard model, etc.) $-71.30 .+$ h Metal-insulator transitions and other electronic transitions

The periodic Anderson model (PAM) and the Hubbard model (HM) are two of the most fundamental models in condensed matter physics. Despite the simplicity of their Hamiltonians, the many body nature of these models results in a complicated correlated electron problem which does not allow for an exact solution except for the onedimensional HM. Also in infinite dimensions [1,2], where both models map onto a single impurity Anderson model with different self-consistency conditions, an exact solution is only possible numerically. In a recent paper [3], such a numerical study showed an astonishingly similar behavior of the two models and, in particular, that the PAM exhibits a transition similar to the Mott transition of the HM.

In the present paper we show analytically that under certain assumptions the infinite dimensional PAM and the HM become equivalent in the limit of vanishing quasiparticle weight $Z$. These assumptions are similar in nature to those employed for the self-consistent projective method [4] and the linearized dynamical mean-field theory [5]. Thus, the hitherto numerically found similarity can be understood and the critical Coulomb interaction $U_{c}$ for the Mott transition of one model can be determined from that of the other model. Applying the numerical renormalization group (NRG) at zero temperature $(T=0)$ and the quantum Monte Carlo (QMC) technique at finite $T$, we investigate to what extent the two underlying assumptions are fulfilled and show, for the first time, that the zero tem-

\footnotetext{
a e-mail: held@physik.uni-augsburg.de
}

perature PAM with nearest neighbor hybridization has a Mott transition with quasiparticle weight $Z \rightarrow 0$ at a finite $U_{c}$, in contrast to the single impurity Anderson model for which $Z \rightarrow 0$ at $U_{c}=\infty$.

The Hamiltonian of the HM reads

$$
H=-t \sum_{\langle i j\rangle \sigma} f_{i \sigma}^{\dagger} f_{j \sigma}+U \sum_{i}\left(n_{i \uparrow}^{f}-\frac{1}{2}\right)\left(n_{i \downarrow}^{f}-\frac{1}{2}\right) .
$$

Here, $f_{i \sigma}^{\dagger}$ and $f_{j \sigma}$ are creation and annihilation operators for an electron with spin $\sigma$ on site $i$ or $j$, respectively, $n_{i \sigma}^{f}=f_{i \sigma}^{\dagger} f_{i \sigma},\langle i j\rangle$ denotes the sum over nearest neighbors, and $t$ the hopping amplitude between them. We use the symbol $f$ for the electrons of the HM since the equivalence of these and the $f$-electrons of the PAM will be reported in this paper. The PAM consists of a band of conducting electrons ( $d$-electrons) and interacting $f$-electrons. Both are coupled via the hybridization $V_{i j}$ :

$$
\begin{aligned}
H= & -t \sum_{\langle i j\rangle \sigma} d_{i \sigma}^{\dagger} d_{j \sigma}+\sum_{i j \sigma} V_{i j}\left(d_{i \sigma}^{\dagger} f_{j \sigma}+\text { h.c. }\right) \\
& +U \sum_{i}\left(n_{i \uparrow}^{f}-\frac{1}{2}\right)\left(n_{i \downarrow}^{f}-\frac{1}{2}\right) .
\end{aligned}
$$

We only consider the particle-hole symmetric case $(\mu=0$ in this form for a symmetric non-interacting density of states (DOS). In infinite dimensions or with the number of nearest neighbors $\mathcal{Z} \rightarrow \infty$, a non-trivial scaling of the kinetic energy is obtained by $t=t^{*} / \sqrt{\mathcal{Z}}$. In the following, 
$t^{*} \equiv 1$ sets the energy scale. We consider two different kinds of hybridizations: (i) a nearest neighbor hybridization $V_{i j}=t_{d f} / \sqrt{\mathcal{Z}}$ for nearest neighbors $i$ and $j$ which is zero otherwise [6] and (ii) an on site hybridization with $V_{i i}=t_{d f}$ and zero otherwise [7].

Within dynamical mean field theory (DMFT) [1,2], which becomes exact in infinite dimensions, the HM and the PAM map onto the same single site problem (which depends on the Green function $G_{f}$, self-energy $\Sigma_{f}, T$, and $U)$ but different self-consistency conditions. For the HM this self-consistency condition at frequency $\omega$ is given by

$$
G_{f}(\omega)=\int d \epsilon \frac{N(\epsilon)}{\omega-\Sigma_{f}(\omega)-\epsilon},
$$

where $N(\epsilon)$ is the non-interacting DOS. In the case of the PAM, the $d$-electrons can be integrated out since they enter only quadratically in the Hamiltonian and the effective action. This results in an effective $f$-electron problem with a self-consistency condition that reads

$$
G_{f}(\omega)=\int d \epsilon \frac{N(\epsilon)}{\omega-\Sigma_{f}(\omega)-t_{d f}^{2} \epsilon^{2} /(\omega-\epsilon)}
$$

for the PAM with nearest neighbor hybridization and

$$
G_{f}(\omega)=\int d \epsilon \frac{N(\epsilon)}{\omega-\Sigma_{f}(\omega)-t_{d f}^{2} /(\omega-\epsilon)}
$$

for the PAM with on site hybridization, where $N(\epsilon)$ is the free $d$-electron DOS. Note, that the effective one-particle potential of the PAM $(\propto 1 /(\omega-\epsilon))$ is frequency dependent, i.e., retarded, due to the fact that the electrons may move from the $f$-orbitals to the $d$-band and return at a later time. The main difference between nearest neighbor [Eq.(4)] and on site hybridization [Eq.(5)] is that the former describes metallic f-electrons at $U=0$ and within a Fermi liquid phase while the latter describes Kondoinsulating f-electrons, i.e., a gapped f-electron quasiparticle peak induced by the hybridization.

The equivalence of the PAM and the HM (at the Mott transition $Z \rightarrow 0$ ) is shown on the basis of two assumptions: (i) that the metallic phase of the $f$-electrons may be described by Fermi liquid theory at low energies and (ii) that the remaining spectral weight of $1-Z$ is contained in two Hubbard bands centered around $\pm U / 2$ and, in particular, that differences in the internal structure of these bands have no influence on the low-energy physics. Assumption (ii) is certainly only fulfilled approximately and becomes justified if the high energy features are well separated from the low-energy features \&]. For a detailed discussion on this assumption see Sec. 2 of [5].

With assumption (i) and $Z=\left(1-\partial \Sigma_{f} /\left.\partial \omega\right|_{\omega=0}\right)^{-1}$ the low-frequency self-consistency condition for the PAM with nearest neighbor hybridization is given by

$$
\begin{aligned}
G_{f}(\omega) & =\int d \epsilon \frac{Z N(\epsilon)}{\omega-Z t_{d f}^{2} \epsilon^{2} /(\omega-\epsilon)} \\
& =\int d \epsilon \frac{Z N(\epsilon)}{\omega-\epsilon / 2+\sqrt{1+4 Z t_{d f}^{2}} \epsilon / 2} \frac{\sqrt{1+4 Z t_{d f}^{2}}+1}{2 \sqrt{1+4 Z t_{d f}^{2}}}
\end{aligned}
$$

$$
+\frac{Z N(\epsilon)}{\omega-\epsilon / 2-\sqrt{1+4 Z t_{d f}^{2}} \epsilon / 2} \frac{\sqrt{1+4 Z t_{d f}^{2}}-1}{2 \sqrt{1+4 Z t_{d f}^{2}}} .
$$

With the partial fraction decomposition above and a variable transformation $y=\frac{1}{2 Z}\left(1 \mp \sqrt{1+4 Z t_{d f}^{2}}\right) \epsilon$ for the two terms of Eqn. (7) one obtains

$$
G_{f}(\omega)=\int d y \frac{Z \tilde{N}(y)}{\omega-Z y}
$$

which is just the low-frequency self-consistency condition of the HM within a Fermi liquid phase. However, with a DOS which depends on $Z$ (which is itself a function of $U$ ):

$$
\begin{aligned}
\tilde{N}(y) & =N\left(\frac{2 Z y}{\sqrt{1+4 Z t_{d f}^{2}}-1}\right) \frac{\sqrt{1+4 Z t_{d f}^{2}}+1}{\sqrt{1+4 Z t_{d f}^{2}}-1} \frac{Z}{\sqrt{1+4 Z t_{d f}^{2}}} \\
& +N\left(\frac{2 Z y}{\sqrt{1+4 Z t_{d f}^{2}}+1}\right) \frac{\sqrt{1+4 Z t_{d f}^{2}}-1}{\sqrt{1+4 Z t_{d f}^{2}}+1} \frac{Z}{\sqrt{1+4 Z t_{d f}^{2}}} .(9)
\end{aligned}
$$

In the limit $Z \rightarrow 0, \tilde{N}(y)$ reduces to

$$
\tilde{N}(y) \stackrel{Z \rightarrow 0}{\longrightarrow} N\left(y / t_{d f}^{2}\right) / t_{d f}^{2}+\mathcal{O}\left(Z^{2}\right) .
$$

and becomes, thus, independent of $Z$. Therefore, at $Z \rightarrow$ 0 , the low energy spectral function of the PAM is identical to that of the HM with the DOS of Eq. (10). With assumption (ii), i.e., that differences in the internal structure of the Hubbard bands have no impact on the low-energy physics, the PAM with nearest neighbor hybridization is equivalent to a HM which has the DOS of the PAM's $d$ electrons renormalized by the factor $1 / t_{d f}^{2}$. Thus, the critical Coulomb interaction and temperature for the Mott transition of the PAM can be calculated from that of the HM via

$$
U_{c}^{\mathrm{PAM}}=t_{d f}^{2} U_{c}^{\mathrm{HM}} \quad \text { and } \quad T_{c}^{\mathrm{PAM}}=t_{d f}^{2} T_{c}^{\mathrm{HM}} .
$$

For the PAM with on site hybridization, the same proceeding yields for $Z \rightarrow 0$ :

$$
G_{f}(\omega) \stackrel{Z \rightarrow 0}{\longrightarrow} \int_{-\infty}^{\infty} d y \frac{Z N\left(-t_{d f}^{2} / y\right)}{\omega-Z y} \frac{t_{d f}^{2}}{y^{2}}
$$

Again, this is equivalent to the low-frequency self-consistency condition of a HM. While the PAM with nearest neighbor hybridization (which has metallic $f$-electrons at small $U$ ) maps to a familiar HM, the PAM with on site hybridization (which yields a Kondo insulating $f$ electron system) maps to a rather unusual HM. If the free $d$-electron DOS of the PAM $N(\epsilon)$ is zero for $|\epsilon|>D$, the PAM with on site hybridization is equivalent to a HM with a gap of size $t_{d f}^{2} / D$ in the non-interacting DOS. This reflects the Kondo insulating nature of this model. Furthermore, the DOS of this unusual HM has not a finite bandwidth but tails decaying like $1 / y^{2}$ for large energies. In particular, the standard deviation of this DOS 


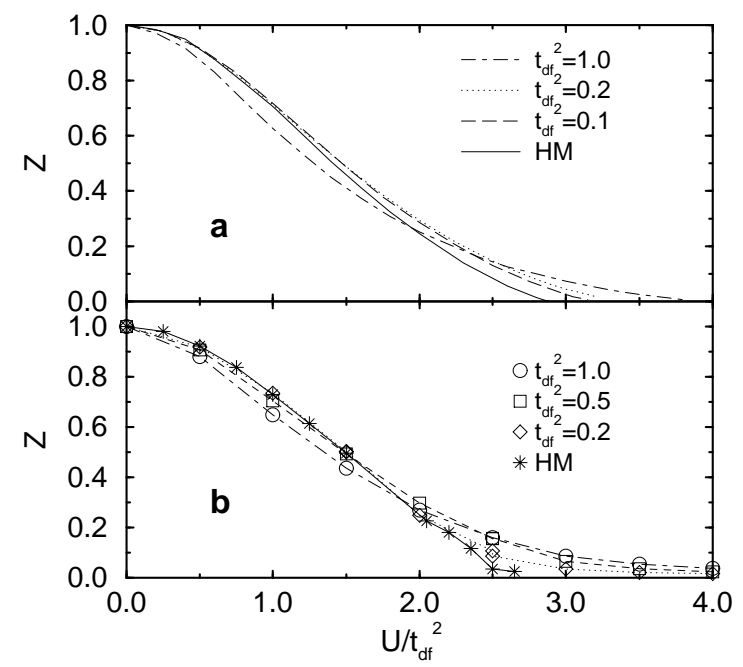

Fig. 1. Quasiparticle weight $Z$ as a function of $U / t_{d f}^{2}$ for the PAM with nearest neighbor hybridization at a) $T=0$ (NRG) and b) $T / t_{d f}^{2}=0.05$ (QMC) in comparison to that of the HM. The QMC data for the HM are partly from 11.

is infinite. For such a DOS, the linearized DMFT [5] predicts a Mott transition at $U_{c}=\infty$ and for the HM with Lorentzian DOS (a similar DOS without gap) it is known that $U_{c}=\infty$ [9]. Thus, at $T=0$ the analytic argument suggests $U_{c}=\infty$ for the PAM with on site hybridization, in agreement with recent NRG results [10]. Nevertheless, at finite $T$ the transition is very similar to that of the HM and the PAM with nearest neighbor hybridization [3]. This is due to the fact that the vanishing of the quasiparticle peak at a fixed finite temperature is unaffected by the very small energy scale present at $T=0$.

With the approximative nature of assumption (ii) in mind we investigate now numerically by NRG [12] and QMC [13] to what extent the derived scaling relations hold. To this end, we calculate $Z$ [14] as a function of $U / t_{d f}^{2}$ for a Bethe lattice. The results are presented in Fig. 1 which shows that there is indeed a Mott transition $Z \rightarrow 0$ at $T=0$ in the PAM with nearest neighbor hybridization. As in the HM [2, 15, 16], the coexistence of two solutions is observed at $T=0$ (Fig. I only contains the metallic solution obtained with increasing $U$ ). Fig. 1 validates, furthermore, that the scaling relation Eqn. (11) holds, at least approximately, even though, the actual values of $U$ differ by a factor of 10, both at zero and finite temperature (at finite temperature, $T / t_{d f}^{2}$ was kept constant according to Eq.(11)).

From Fig. 11 the critical value of $U$ for the Mott transition, i.e., the vanishing of $Z$, is determined $\sqrt{17}$. The results are compared to prediction (11) in Fig. 2. At small $t_{d f}$, the agreement is very good, while there are notable deviations at larger $t_{d f}$. These deviations can be understood from the spectral functions discussed below.

Fig. 3 shows the disappearance of the central quasiparticle peak at the Mott transition in the $f$-electron spectrum of the PAM with nearest neighbor hybridization. At the same time, the $d$-electron spectral function remains finite at the Fermi energy. Thus, despite the Mott transi-

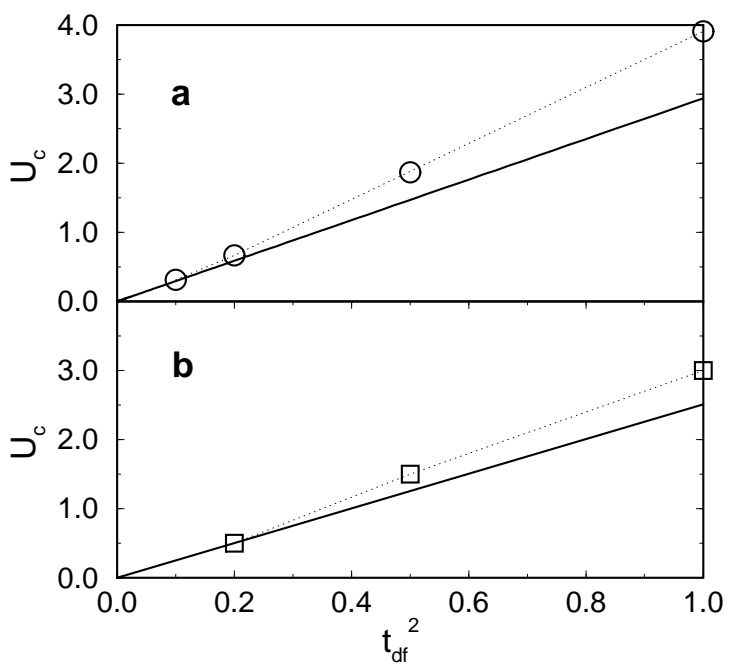

Fig. 2. Critical value $U_{c}$ for the Mott transition of the PAM with nearest neighbor hybridization at a) $T=0$ (circles) and b) $T / t_{d f}^{2}=0.05$ (squares) compared to $t_{d f}^{2} U_{c}^{\mathrm{HM}}$ (solid line) at these temperatures.

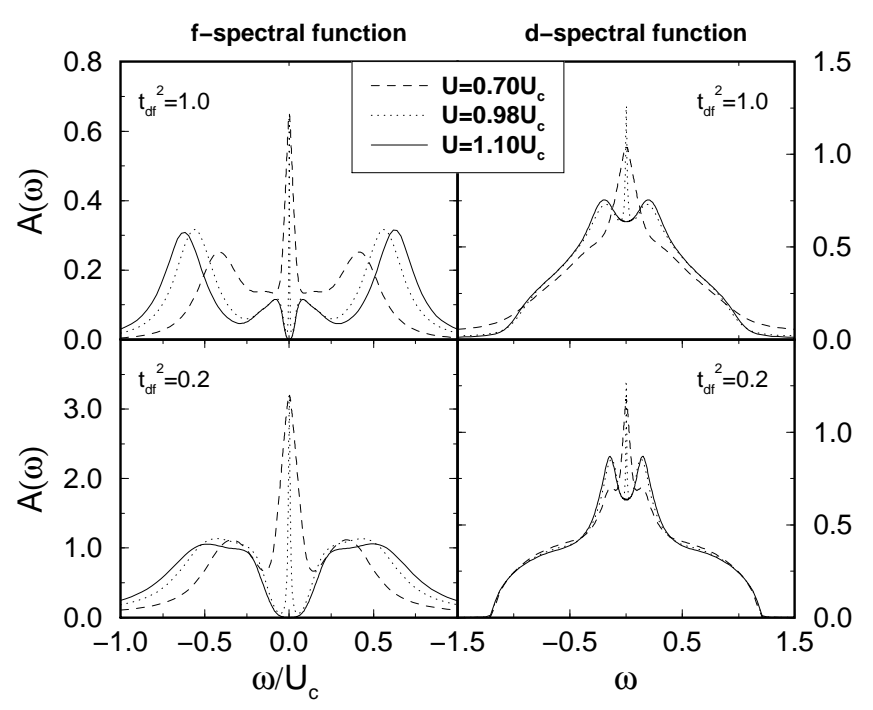

Fig. 3. Spectral function of the $f$ - and $d$-electrons for the PAM with nearest neighbor hybridization at $T=0$ (NRG) and at different values of $U$ close to the Mott transition.

tion of the $f$-electrons the overall system remains metallic. W.r.t. the deviations between $U_{c}$ and prediction (11), one observes that at $t_{d f}^{2}=0.2$ the quasiparticle resonance is well separated from the high-energy Hubbard bands, while at $t_{d f}=1$ there is additional spectral weight very close to the quasiparticle resonance. Thus, assumption (ii) is not a good approximation for larger $t_{d f}$ with the consequence that the analytic calculation based on assumption (ii) is less justified and prediction (11) less accurate. This explains the the $t_{d f}$-dependence of the deviations in Fig.2 which is a priori not clear from the analytic calculation.

Finally, Fig. 4 shows a comparison between the $f$ spectral functions for the periodic Anderson model with 


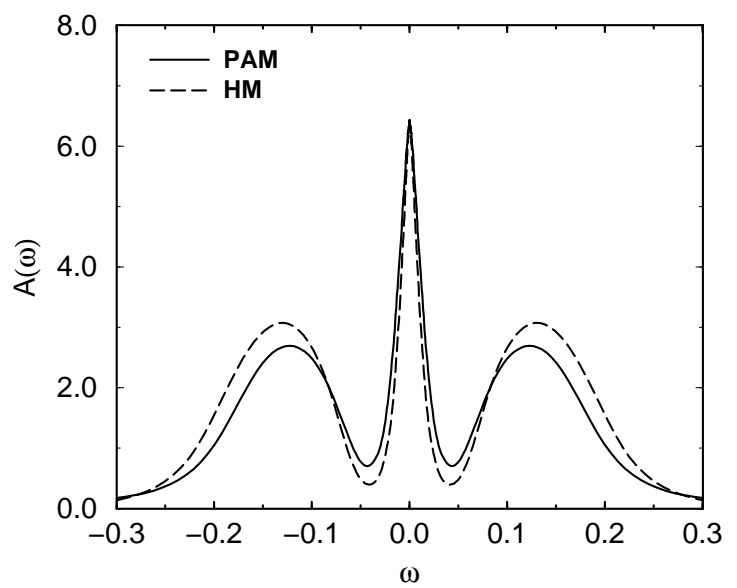

Fig. 4. $f$-spectral functions of the periodic Anderson model with nearest neighbour hybridization compared to the corresponding Hubbard model according to Eq. (10) at $U=0.25$, $t_{d f}^{2}=0.1$, and $T=0$ (NRG).

nearest neighbour hybridization and the Hubbard model. The density of states for the Hubbard model calculation is chosen according to Eq. (10). As expected, the results show a good agreement in the low-frequency part whereas the deviations are more pronounced in the high-frequency regime. The agreement for small $\omega$ is, however, not perfect, even in the limit $Z \rightarrow 0$, as the derivation of Eq. (10) is only approximate.

In conclusion, we showed analytically that the PAM becomes equivalent to the HM at the Mott transition $Z \rightarrow 0$ if (i) the low energy physics of both models is described by Fermi liquid theory and (ii) the high energy features are Hubbard bands which are well separated from the low energy quasiparticle peak. This allows to calculate the critical interaction $U_{c}$ at which $Z \rightarrow 0$ for the PAM from that of the Hubbard model. In particular, the PAM with on-site hybridization maps to a Hubbard model with gapped DOS and Lorentzian tails which suggests $U_{c}=\infty$, while the PAM with nearest neighbor hybridization maps to the Hubbard model with the same DOS as the $d$-electron DOS of the PAM. The latter leads to the scaling relation $U_{c}^{\mathrm{PAM}}=t_{d f}^{2} U_{c}^{\mathrm{HM}}$. Numerical calculations employing NRG and QMC yield that the PAM with nearest neighbor hybridization has indeed a Mott transition at a finite $U_{c}$ and that the above scaling relation is correct for not too large values of $t_{d f}$. The similarity between both models includes the existence of hysteresis for the PAM with nearest neighbor hybridization.

We are grateful to N. Blümer, C. Huscroft, A.K. McMahan, Th. Pruschke, R.T. Scalettar, and D. Vollhardt for valuable discussions. This work was supported in part by the Sonderforschungsbereich 484 of the Deutsche Forschungsgemeinschaft.

\section{References}

1. W. Metzner and D. Vollhardt, Phys. Rev. Lett. 62, 324 (1989); D. Vollhardt, in Correlated Electron Systems, V. J. Emery ed. (World Scientific, Singapore), p. 57 (1993); Th. Pruschke, M. Jarrell, and J. K. Freericks, Adv. Phys. 44, 187 (1995).

2. A. Georges, G. Kotliar, W. Krauth, and M. Rozenberg, Rev. Mod. Phys. 68, 13 (1996).

3. K. Held, C. Huscroft, R.T. Scalettar, and A.K. McMahan, Phys. Rev. Lett. 84, 373 (2000).

4. G. Moeller, Q. Si, G. Kotliar, M. J. Rozenberg, and D. S. Fisher, Phys. Rev. Lett. 74, 2082 (1995).

5. R. Bulla and M. Potthoff, Eur. Phys. J. B 13, 257 (2000).

6. A three-dimensional QMC study of this nearest neighbor hybridization can be found in C. Huscroft, R.T. Scalettar, and A.K. McMahan, Phys. Rev. Lett. 82, 2342 (1999).

7. DMFT studies of this on site hybridization include: M. Jarrell, H. Akhlaghpour, and Th. Pruschke, Phys. Rev. Lett. 70, 1670 (1993); M. Jarrell, Phys. Rev. B 51, 7429 (1995); A.N. Tahvildar-Zadeh et al., Phys. Rev. B 55, 3332 (1997); M. Rozenberg, Phys. Rev. B 52, 7369 (1995). Two-dimensional QMC studies include: J. Bonča and J.E. Gubernatis, Phys. Rev. B 58, 6992 (1998); Y. Zhang and J. Callaway, Phys. Rev. B 38, 641 (1988); M. Vekic et al., Phys. Rev. Lett. 74, 2367 (1995).

8. For the HM, the QMC simulation [11] shows that besides the central resonance there is additional spectral weight at low frequencies, also close to the Mott transition. Thus, at higher temperatures, approximation (ii) is less good than at $T=0$ where NRG results 15] show a more complete separation of the central resonance from the rest of the spectrum.

9. A. Georges, G. Kotliar, and Q. Si, Int. J. Mod. Phys. B 6, 705 (1992).

10. Th. Pruschke, private communication.

11. J. Schlipf et al., Phys. Rev. Lett. 82, 4890 (1999).

12. For the NRG algorithm see: K.G. Wilson, Rev. Mod. Phys. 47, 773 (1975); H.R. Krishna-murthy, J.W. Wilkins, and K.G. Wilson, Phys. Rev. B 21, 1003 (1980); R. Bulla, preprint cond-mat/0003377 (2000).

13. For the QMC algorithm see: J. E. Hirsch and R. M. Fye, Phys. Rev. Lett. 56, 2521 (1986); M. Jarrell, in Numerical Methods for Lattice Quantum Many-Body Problems, D. Scalapino ed., (Addison Wesley), (1997); M. Ulmke Phasenübergänge in stark korrelierten Elektronensystemen, Ph.D. thesis RWTH Aachen (1995) [ISSN 0944-2951].

14. To calculate $Z$ within NRG, $Z=\left(1-\partial \operatorname{Re} \Sigma(\omega) /\left.\partial \omega\right|_{\omega=0}\right)^{-1}$ is directly employed. Within QMC, $Z$ is determined via the finite differential quotient $\left(1-\operatorname{Im} \Sigma\left(i \omega_{0}\right) / \omega_{0}\right)^{-1}$ at the lowest Matsubara frequency $\omega_{0}$. The latter gives a small $Z$ at finite temperatures, even after the disappearance of the quasiparticle resonance. The QMC time discretization $\Delta \tau$ was $\Delta \tau=0.25,0.333$, and 0.5 for $t_{d f}^{2}=1,0.5$, and 0.2 , respectively.

15. R. Bulla, Phys. Rev. Lett. 83, 136 (1999).

16. M. J. Rozenberg, R. Chitra, and G. Kotliar, Phys. Rev. Lett. 83, 3498 (1999).

17. To determine $U_{c}$ within $\mathrm{QMC}$, we decide on metal or insulator via the approximate criterion. $\operatorname{Im} \Sigma\left(\omega_{0}\right)<\operatorname{Im} \Sigma\left(\omega_{1}\right)$ : indicates $\Sigma(\omega) \stackrel{\omega \rightarrow 0}{\longrightarrow}$ 0, i.e, a metal; $\operatorname{Im} \Sigma\left(\omega_{0}\right)<\operatorname{Im} \Sigma\left(\omega_{1}\right)$ : insulator. 